\title{
The binary supermassive black hole conjecture for jetted active galactic nuclei
}

\author{
Stefano Ciprini* ${ }^{* \dagger}$ \\ Space Science Data Center, Agenzia Spaziale Italiana (SSDC-ASI), I-00133, Roma, Italy \\ Istituto Nazionale di Fisica Nucleare (INFN), Sezione di Roma Tor Vergata, I-00133, Roma, Italy \\ E-mail: stefano.cipriniessdc.asi.it
}

\begin{abstract}
Advances in the knowledge of the universal accretion phenomenon and jet physics, are important not only for astrophysics of stellar-size systems in our Galaxy like cataclysmic variable stars and active galactic nuclei (AGNs), but could be able to contribute to some contemporary multimessenger and astro-particle research topics. Astrophysical accretion and binary systems are undoubtedly useful, especially in the case of outflows and production of jets, to understand the role of gravitation in producing high-energy, X-ray and gamma-ray, emission, gravitational waves, accelerated very/ultra-high-energy neutrinos and cosmic rays. In particular close and spatiallyunresolved gravitationally bound binaries of supermassive black holes (SMBHs) in AGN are expected to induce cyclical modulations and outbursts in the emitted electromagnetic flux and to produce micro/nano-Hz frequency gravitational waves. The first discovery of a very high energy neutrino from a flaring $\mathrm{GeV}$ gamma-ray blazar and theoretical predictions about the possibility of accreting black holes with gravitationally bound axion-like particle clouds subject to superradiance, are pointing out AGN and blazars as cosmic beacons able to represent also optimal cosmic laboratories for gravitation and high-energy particle physics. In this frame some developments on the last observational results about a famous radio/gamma-loud, jetted, blazar OJ 287, that is expected to harbor a binary SMBH system are here summarized.
\end{abstract}

Accretion Processes in Cosmic Sources - II - APCS2018

3-8 September 2018

Saint Petersburg, Russian Federation

\footnotetext{
* Speaker.

$\dagger$ on behalf of the international observing monitoring program and collaboration devoted to OJ 287 during 2010s (effort co-led mainly by M. J. Valtonen, S. Zola, A. Gopakumar, R. Hudec, M. Kidger, S. Laine, L. Dey, P. Pihajoki, A. C. Gupta, A. Goyal, SC, H. Lehto, S. Komossa, A. Berdyugin, S. Mikkola, K. Matsumoto, K. Sadakane, V. Piirola, K. Nilsson, A. Sillanpää, M. Ostrowski, A. Gopal-Krishna, G. Bhatta, Ł. Stawarz, D. Reichart, K. Gazeas and many other).
} 


\section{Introduction: blazar laboratories for astrophysics}

Almost all galaxies contain a massive black hole and about $99 \%$ of them are, not-completely, silent like our Milky Way Galaxy. About $1 \%$ of the galaxies in the Universe is active and most of them are radio-quiet active galactic nuclei (AGN). About $0.1 \%$ are radio loud AGN and endowed with jets, mostly visible at radio wavebands. Quasars, blazars, radio galaxies and other types of radio-loud AGN can be defined as the most powerful electromagnetic beacons and particle beams of the Universe. The great power in AGN is driven by accreting matter onto a super-massive black hole (SMBH), and part of this accretion energy fuels a relativistic jet (particles and energy). Among the other some recent reviews can be found in [36, 12, 30, 47, 20].

In blazars the magnetized plasma jet dominated the observed energy output and it points within about 10 degrees of the Earth's line of sight, strengthening macroscopic Special Relativity cinematic effects like the relativistic bulk Doppler beaming of the radiation, the shifting of emission to higher frequencies, and the shortening of time-scales of variability [16, 47]. As a result, blazars appear to be the most luminous cosmic objects that persist for more than a few minutes, and if we could see the sky with eyes sensitive to either microwave band or gamma-ray radiation, the sky outside the Milky Way band, would shine with blazars, rather than planets and stars. This powerful radiation is observed to change in brightness on time-scales as short as minutes, yet there is strong evidence that this rapid electromagnetic flux variability often occurs one or more parsecs from the black hole, where longer time-scales would normally be expected.

Blazars are powered by SMBHs masses of order 0.1-10 billion solar masses, accreting material from their environment at rates measured in solar masses per year, and generally producing a pair of such jets launched in opposite directions. Jets are highly collimated relativistic outflows, i.e. relativistic beams, with bulk Lorentz factors in the range of about 5 and 40. At radio wavebands they are characterized by a compact unresolved radio core, a flat and inverted radio continuum spectra, large radio brightness temperatures and superluminal motion at radio-band scales observed with very long baseline interferometry (VLBI).

Blazar is mostly a phenomenological term (observational characteristics) and represents broad active accretion systems and relativistic particle accelerators [36, 12,30]. Usually they can be powerful, $\mathrm{MeV}-\mathrm{GeV}$ photon energy, high-energy gamma-ray sources, with gamma-ray emission dominating the multi-frequency bolometric radiative power output, and making them optimal probes to explore the distant Universe at cosmological scales. Blazars show rapid, irregular and strong electromagnetic flux variability on very different time scales and at all the energy bands (radio to gamma rays), and a relatively high degree of optical and radio polarization. Their accretion disks produces optical, UV and X-ray emission via various thermal processes. The subfamily of the flat spectrum radio quasars (FSRQs) displays bright broad emission lines in optical and UV wavebands, sometimes an evident continuum blue bump in the spectral energy distribution by the accretion disk, multi-temperature disk emission, non-thermal broad spectral energy distribution (SED) components peaking at IR and hard $\mathrm{X}-\mathrm{ray} / \mathrm{MeV}$ energy bands, a high bolometric luminosity $\left(L>10^{48} \mathrm{erg} \mathrm{s}^{1}\right)$ and redshifts values $z>1$. The subfamily of BL Lacertae objects (BL Lacs) displays weak emission lines (equivalent width $<5$ ), little or no evidence of disk or emission lines in optical and UV bands, non-thermal SED peaks placed at UV/soft X-rays and GeV energy bands, lower bolometric luminosity $\left(L<10^{45} \mathrm{erg} \mathrm{s}^{1}\right)$ and and redshifts values $z<1$. These also displays 


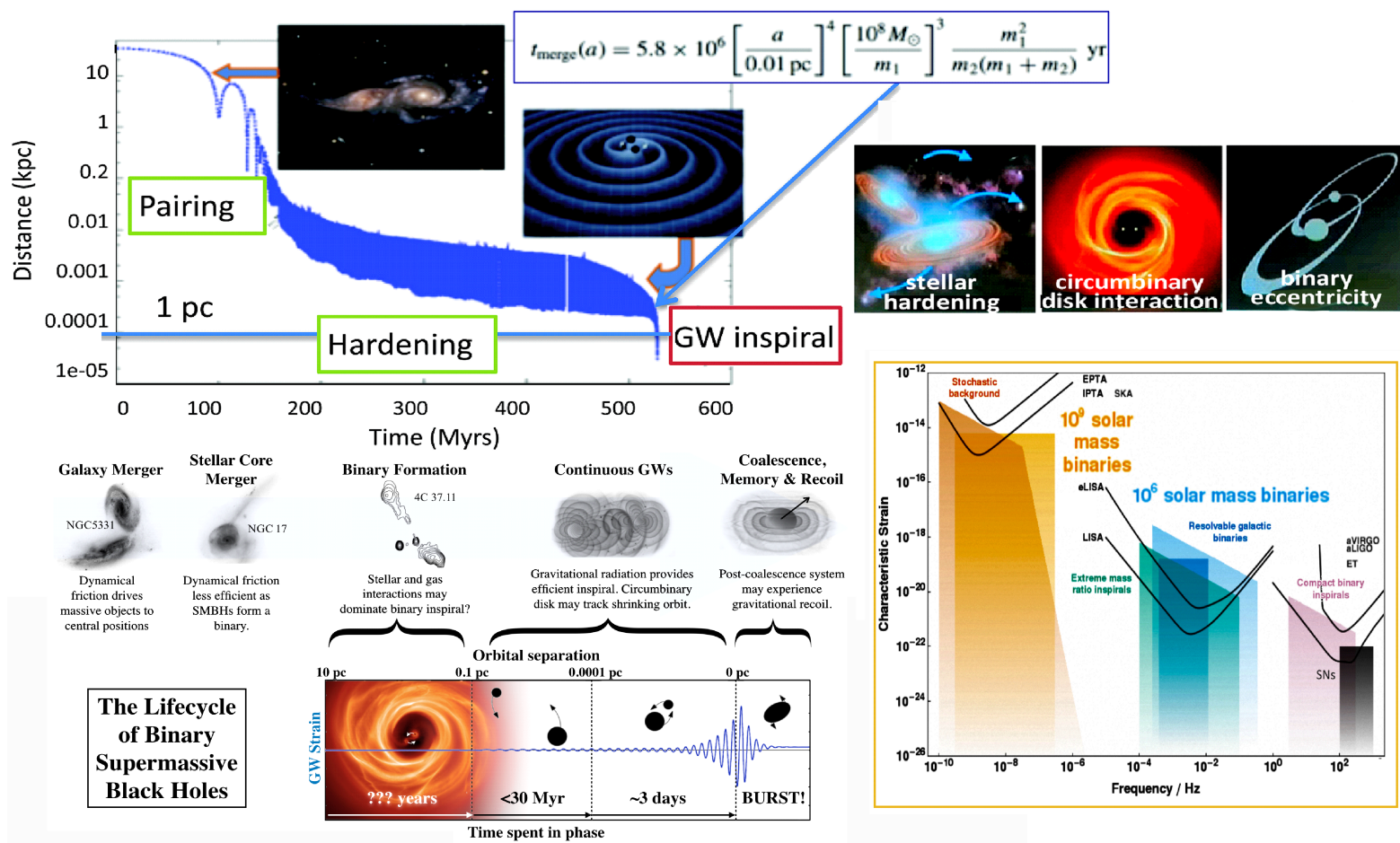

Figure 1: There is little evidence for widespread binary SMBHs, hence they need to merge rather efficiently. Merger is a natural way of producing SMBHs from smaller seeds, and timescale from two galaxy merger to their central SMBH merger is in the range of $10^{8}-10^{9}$ years. Future gravitational astronomy with ultra-low frequency GWs will open a new window on structure formation and galaxy mergers, direct detection of coalescing binary SMBHs, high-precision measurements of SMBHs masses and spins, with constraints on SMBHs formation and evolution.

more turbulent and less powerful radio jets when their SED is peaked at higher energies.

Blazars are variable in emission lines strength, continuum flux brightness, polarization, spectral hardness. Variability over all energy bands (radio to $\mathrm{GeV}-\mathrm{TeV}$ gamma rays) and time scales is a defining property of blazars and also a promise to understand their physics. They are interesting for topics like accreting black hole theories, black-hole and accretion disk relation, accretion and jet physics connection, emission effects on host galaxies and galaxy clusters. They are also useful in probing the extragalactic background light (EBL). AGN unified theoretical and phenomenological models predict that the orientation with observer line-of-sight determines source properties and families (e.g. radio galaxies vs blazars), this joined to other factors and physical properties like the accretion rate, the radio loudness, the SMBH mass and spin, the host galaxy properties $[36,47]$.

For the high-energy SED component emission mechanisms like leptonic processes (e.g. electrons, positrons involving inverse-Compton scattering of synchrotron or external photons and pairs production) and hadronic processes (proton synchrotron emission, hadron-nuclear process with pion decay, photo-hadronic process with photo-pion production) are mostly involved. In hadronic models, both primary electrons and protons are considered to be accelerated to ultrarelativistic energies, with protons exceeding the threshold for photo-pion production on the soft photon field in the emission region, and in all these hadronic models the emission of very-high-energy neutrinos is foreseen. The study of emission location in a relevant portion of cases can be constrained for simplest leptonic models based on a single zone of high-energy emission, where opacity effects 
and energy-dependent photospheres have to be taken into account. The particle acceleration mechanisms are centered on ultrarelativistic shocks physics, turbulence models, magnetic reconnection, pair producion and the Blandford-Znajek mechanism for the extraction of energy from a rotating SMBH. Crucial aspects to understand blazars are also related to jet composition (Poynting flux, leptons, hadrons, ions) and jet confinement (external pressure and magnetic stresses). Accretion processes, merging and stellar captures are very important for the mass growing and spin evolution and history of SMBHs.

SMBH and disk accretion processes, the complex high-energy plasma interactions, the relativistic shock waves, and other physical processes of blazars, are difficult to be studied in laboratories or even in the solar system, where in situ measurements are possible with space probes. Blazars have led to a golden age of space-borne and ground-based astronomical instruments and observational programs. These include time-dependent SED and multifreqeuncy light curves measurements, VLBI techniques that produces microwave images of jets on scales measured in microarcseconds revealing superluminal motion of components in the jet and, recently, the first and direct millimeter image of the M 87 SMBH from the Event Horizon Telescope (EHT). In addition polarization monitoring at radio to optical wavelengths and the next, incoming, X-ray polarization observation by space telescopes like IXPE and eXTP.

Some of the still controversial astrophysical questions regarding blazars are, among the other:

- underlying physics for the variability phenomena at all the various wavebands;

- radio-structure (VLBI) and polarization related to X-ray and gamma-ray flux data, and the relation between the compact radio-emitting features and the high-energy emission;

- physics of sudden emission flares across a wide, or mysteriously, sometimes rather narrowfrequency range of the electromagnetic spectrum;

- emission region location and radiative processes;

- nature and physics of their relativistic jets;

- accretion, variability mechanisms, particles composition and acceleration mechanisms;

- disk-jet connection and accretion-jet-structure physics;

- paradigms to understand the different object populations and their evolution with cosmological distance and time.

Blazars remain both surprising and enigmatic in many ways and after about half a century of effort, astrophysicists continue to struggle to explain the phenomena associated with them, suggesting us that these are really rich and complete physics cosmic laboratories.

\section{Multi-messenger astro-particle physics with AGN}

Advances in the knowledge of the universal accretion phenomenon, also in related jet astrophysics studies for the minority of the population of jetted sources, both in stellar systems like cataclysmic variables, novae stars, young stellar objects, T-Tauri stars, low-mass binaries stars, 
high-mass X-ray binaries stars, soft X-ray/gamma-ray repeaters, magnetars, and in galaxy-sized objects like AGN, can give a contribute also in astro-multi-messenger/multi-particle physics (examples in Fig. 2 and Fig. 3). The study of such accreting objects of classical astronomy, the binary dynamics, the fast extensive energy release, the accretion cycles and variability mechanisms, the study and modeling of accretion disks, tori and jets is useful to better understand:

- the new role of gravitation, emerged since '90s, in producing high-energy, $\mathrm{X}$-ray and $\mathrm{MeV}$ $\mathrm{GeV}$ and $\mathrm{TeV}$ energy gamma-ray radiation;

- the hypotheses based on unresolved and close $(\lesssim 0.1 \mathrm{pc})$ binaries of supermassive black holes (SMBHs) at the center of AGN, producing quasi-periodic variability signatures in the observed electromagnetic flux, helical and distorted pc-scale radio jets with precession, re-

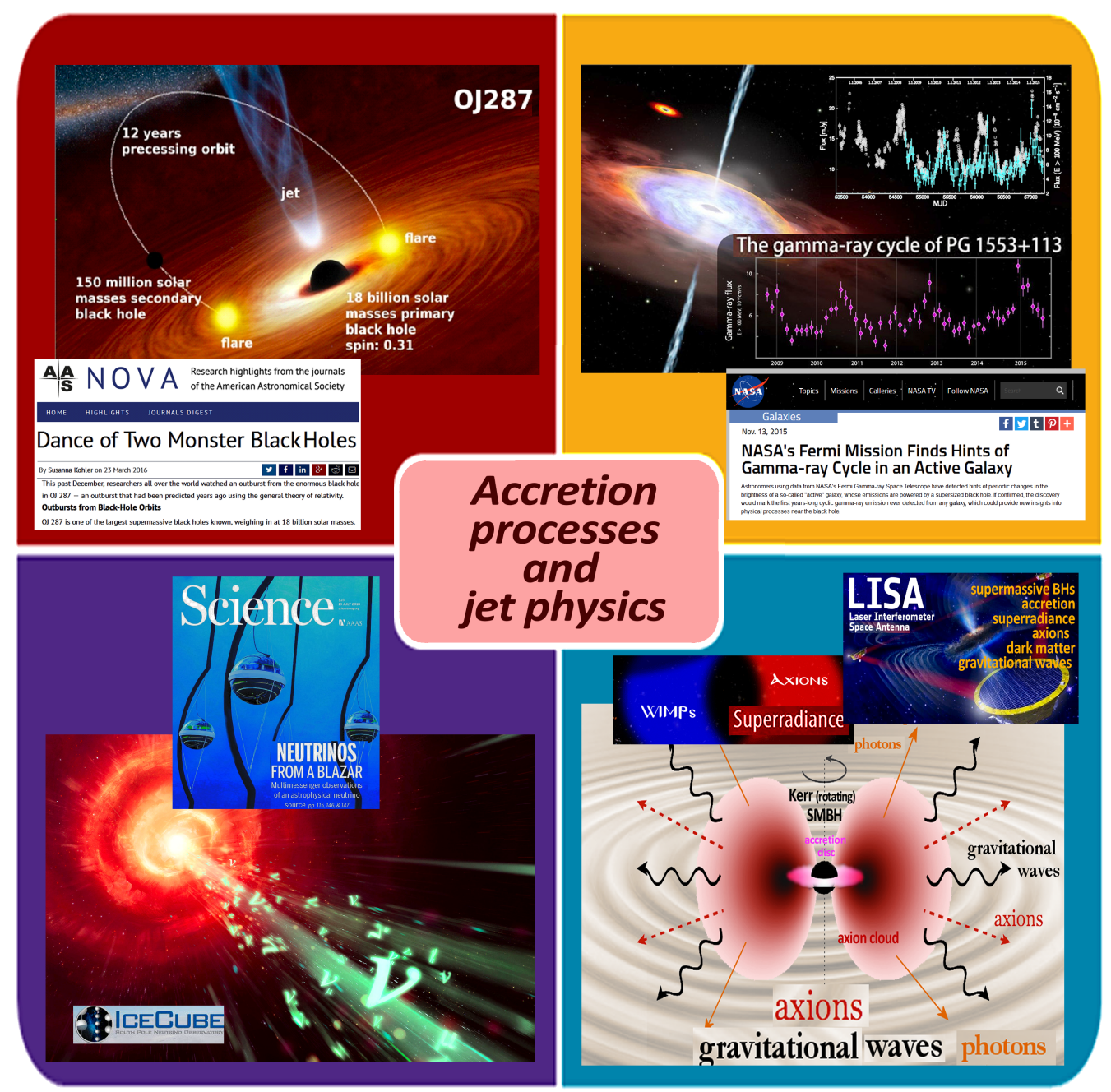

Figure 2: The understand of the universal phenomenon of classic astronomy, the accretion in jetted sources, can be considered a key to understand also postulated binary SMBHs blazar systems where, in case, strongfield GR effects are present, like the two examples reported in the figure (OJ 287 and PG 1553+113 [48, 2]), the possible production of very/ultra high energy neutrinos and cosmic rays in jets of gamma-ray blazars [1], and the theoretical and very-speculative possibility of ALPs particles bounded around the gravitational field of a SMBH producing a GWs superradiance $[3,7]$. 
orientation and X-shape morphologies [29], double-peaked broad emission lines [57], some tidal disruption events [19], post-merging recoils and other more exotic ones;

- the very-low, micro/nano-Hz, frequency gravitational waves produced by close inspiral SMBH binaries (Fig. 1);

- the very/ultra high energy neutrinos and ultra-high-energy (UHE) cosmic rays accelerated in the relativistic jets of gamma-ray blazars and possibly coincident in time-space with blazar's gamma-ray flares, this after the first discovery announced on July 2018 by the IceCube experiment and the Fermi LAT of a $290 \mathrm{TeV}$ energy neutrino (IC 170922A) coincident with a high gamma-ray state of the blazar TXS 0506+056 ( $\mathrm{z}=0.3365)$;

- also, perhaps, the role of accreting black holes serving as hypothetical laboratories for gravitationally bound axion-like particle (ALP) clouds, a viable dark matter candidate, and the related peculiar gravitational wave emission.

Coalescing binary SMBHs are the loudest sources of very-low frequency ( $\mu \mathrm{Hz}$ to $\mathrm{nHz}$ ) gravitational waves (GWs) in the Universe (Fig. 1). Subsequent GW recoil has potential electromagnetic astrophysical implications as the post-merger SMBH can oscillate or even escape. Binary intermediate-mass black hole (IMBH) binaries with $\mathrm{BH}$ masses between $10^{4}$ and $10^{7}$ solar masses and extreme or intermediate-mass ratio inspirals (EMRI/IMRI) are expected to be detected as GW emitters by the future space experiment Laser Interferometer Space Antenna (LISA). LISA is expected to be able to explore, for the first time, the low-mass end of the SMBHs population at cosmic times as early as redshift $>8$. The domain of ultra-low $\mathrm{GW}$ frequencies $(\mathrm{nHz})$ is probed by Pulsar Timing Arrays, PTAs (for binary SMBHs with masses $>10^{7}$ solar masses). PTAs have started to place constraints on galaxy merger history from limits on the stochastic GW background, as the $\mathrm{nHz}$ frequency regime is also the superposition of signals coming from many stationary sources. Future LISA and SKA (PTAs) experiments will provide new possibilities for future GW astronomy, opening new windows on structure formation, galaxy mergers, high-precision measurements of SMBHs masses and spins, this beyond the direct detection of coalescing and merging binary SMBHs, putting crucial constraints on SMBHs formation and evolution.

A possibility given by some recent theoretical physics models, SMBHs and gravitational waves (with chances for coupled electromagnetic signals) may tell us also something useful for dark matter search and physics beyond the Standard Model of fundamental particle physics. The quantum chromo dynamics (QCD) axion is one of the best hypothetical particle motivated beyond standard model of particle physics, solving the strong-CP problem by making the QCD theta angle a dynamical field. It is a Pseudo-Goldstone boson with mass and couplings fixed by the decay constant, very weakly interacting and with a large Compton wavelength. Axion Like Particles (ALPs) are hypothetical and a more general and richer frame, and BHs could be used as natural cosmic ALPs particles detectors, because the BH size is similar to the axion Compton wavelength, and the strong gravity regime. The hairy-axionic black hole would be a "Gravitational Atom", in analogy with the quantum levels of the Hydrogen atom. ALPs clouds gravitationally bound around a BH can occupy quantum states characterized by the usual atomic quantum numbers, by a sort of fine-structure constant and quantized energy levels [3, 7]. Spinning, Kerr, BHs are unstable against 
gravitationally bound ultralight boson fields like that of the ALPs clouds, through superradiance phenomenon, and form a continuous GW source with frequency given by the specific particle mass (Fig. 2).

There is a variety of models for production of astrophysical very/ultra high-energy (VHE/UHE) neutrinos: 1) cosmic-ray accelerator models in sources like AGNs, GRBs, magnetars, high-mass binaries, microquasars and young stellar objects; 2) cosmic-ray reservoir models in sources like star forming region cocoons and supernova renmants, starburst galaxies, galaxy groups and clusters. In the first case cosmic neutrinos are produced within the cosmic-ray source and mesons are typically produced by interactions of cosmic rays with ambient photons. In the second scenario neutrinos are produced while they are confined within the environment surrounding the cosmic-ray source and are produced by inelastic hadro-nuclear collisions. Very high energy processes that involve neutrinos are hadro-nuclear and photo-hadronic processes, but multi-messenger relationships may be more complicated, also considering that electromagnetic radiation (gamma rays) are not exclusively produced in hadronic processes in the source. A first hint of spatial-temporal correlation of VHE

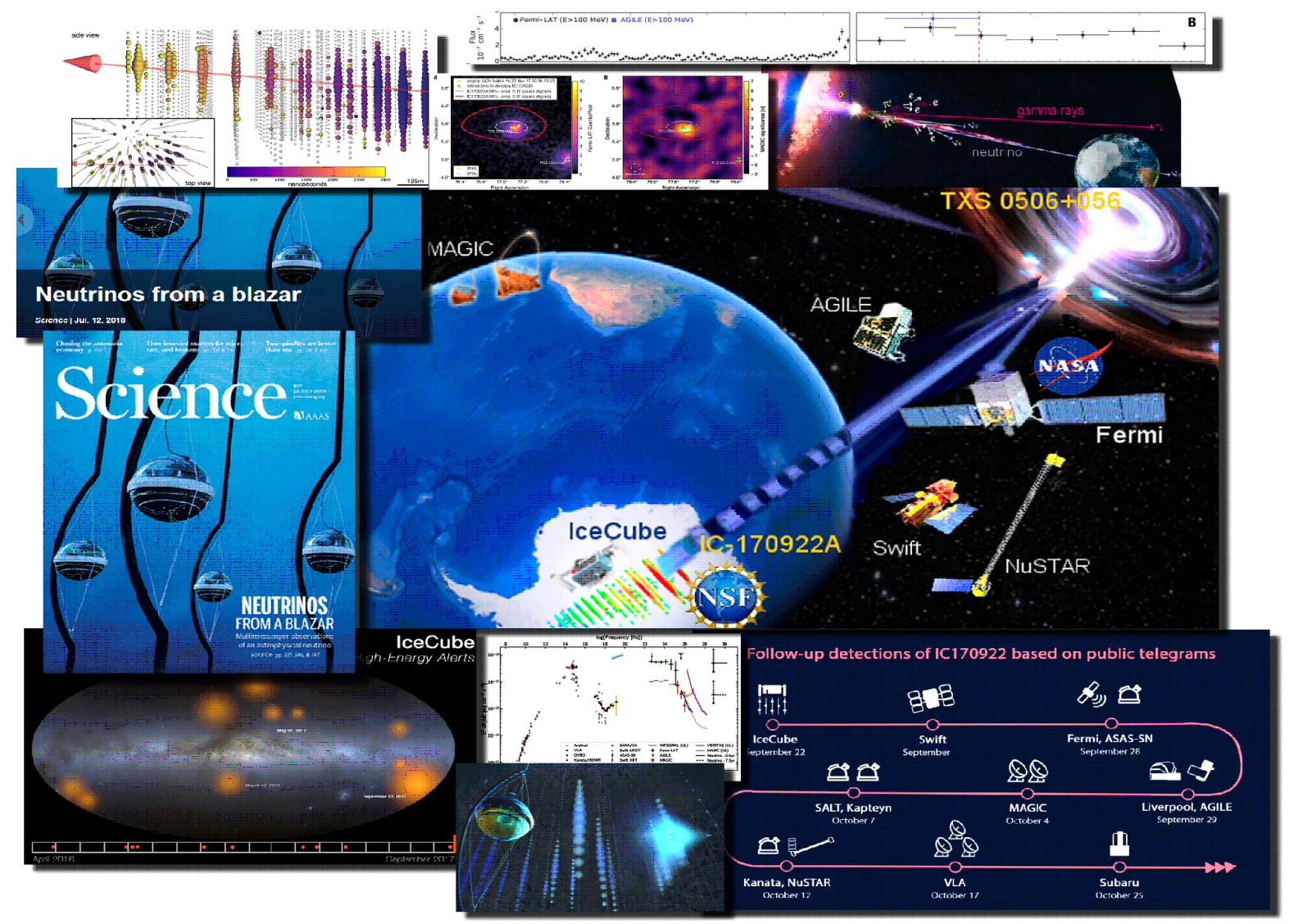

Figure 3: Collage of some figures that accompanied the release of the multimessenger observations of the flaring gamma-ray blazar TXS 0506+056 ( $\mathrm{z}=0.3365$, also known as MG1 J050927+0541, RX J0509.3+054, ZS 0506+056) coincident with high-energy neutrino of IceCube IC170922A. IceCube, Fermi-LAT, MAGIC, AGILE, ASAS-SN, HAWC, H.E.S.S., INTEGRAL, Kanata, Kiso, Kapteyn, Liverpool Telescope, Subaru, Swift/NuSTAR, VERITAS, VLA/17B-403 Collaborations and Teams have participated to this multimessenger analysis and paper [1] announced on July 12, 2018 with a press conference at US National Science Fondation NSF in Washington. The IceCube experiment is placed at the Amundsen-Scott South Pole Station in Antarctica. 
IceCube neutrino with Fermi LAT observed blazar gamma-ray flares is represented by flat spectrum radio quasar PKS 1424-41 with consistence with the $2 \mathrm{PeV}$ "Big Bird" neutrino event detected by IceCube on December 2012 [24], even if there is a not negligible probability of random coincidence. In April 2016, the IceCube experiment at the Amundsen-Scott South Pole Station, started to release real-time alerts of detections of very-high-energy $(>100 \mathrm{TeV})$ cosmic neutrinos events. The IceCube event IC170922A (a $290 \mathrm{TeV}$ energy neutrino) had a temporal, spatial and energy coincidence with a gamma-ray flaring phase for a Fermi LAT blazar: TXS 0506+056 (z=0.3365, also known as MG1 J050927+0541, RX J0509.3+054, ZS 0506+056) [1]. Chance correlation of this neutrino with the electromagnetic high-energy flare of TXS 0506+056 is statistically disfavored at the level of 3 sigma in any of the evaluated models associating neutrino and gamma-ray production. Many follow-up HESS, AGILE, Swift, and MAGIC (that detected it at VHE gammarays above $200 \mathrm{GeV}$ ) were performed corroborating this discovery. The newborn multi-messenger astro-particle physics confirms the close relations among the different cosmic messengers, and multi-messenger plus time-domain studies are a promising path to reveal the origin of energetic neutrinos and cosmic rays.

\section{SMBHs in AGN}

Supermassive black holes (SMBHs) are a ubiquitous component of the nuclei of galaxies and AGN. Following the merger of two massive galaxies, a SMBH binary will form, shrink due to stellar or gas dynamical processes and ultimately coalesce by emitting a burst of very low frequency gravitational waves $[4,10,26,25]$. Simulations of the merger between two disk galaxies show that during the interaction, tidal forces tear the galactic disks apart, generating spectacular tidal tails and
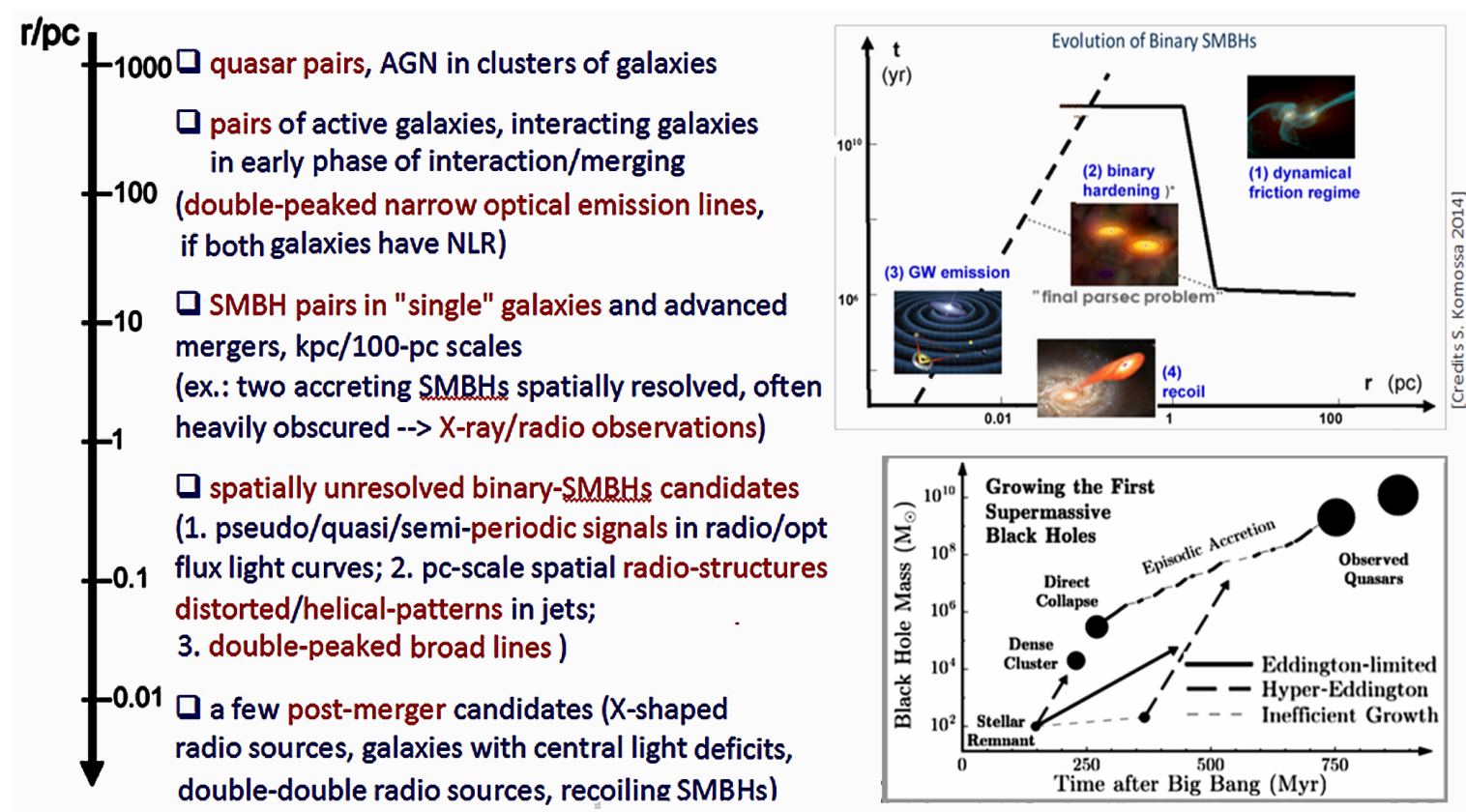

Figure 4: Left panel: table of observational evidence for SMBH pairs and gravitationally-bound binary SMBH systems as depending on the couple/binary separation distance $[4,10]$. Right panels: main physical stages of the evolution of binary SMBHs, and growing scheme of the primordial SMBHs of the Universe (adapted from [25, 44]). 
plumes, and two SMBHs form an eccentric binary in the disk in less than a million years as a result of the gravitational drag from the gas rather than from the stars [32,33]. Galaxy merger is a natural way for the production of SMBHs from smaller seeds (Fig. 1). The merger of two galaxies creates a common nucleus and the dynamical friction rapidly brings the two SMBH together to form a gravitationally bound binary (separation $r \simeq 10 \mathrm{pc}$ ). Three-body interaction of binary with stars of galactic nucleus ejects most stars from the vicinity of the binary by the slingshot effect [34], and a sort of mass deficit is created: the binary becomes "hard" ( $r \simeq 1 \mathrm{pc})$.

The binary SMBH further shrinks by scattering off stars that continue to flow into the loss cone, due to two-body relaxation or other factors. As the separation reaches $0.01 \mathrm{pc}$, gravitational wave emission becomes dominant in carrying away the energy from the system and reaching a few Schwarzschild radii ( $\simeq 10^{-5}$ pc) the binary SMBH finally merges (Fig. 4). Observational evidence is important to confirm the solving of the theoretical final parsec "problem" in General Relativity (GR) solved, for example by some non spherical geometries [54] or by the binary inward migration induced by interaction with the accretion disk [22].

Evolution stages of the SMBH binary and their non-self-gravitating circumbinary accretion disks are studied in semi-analytically and numerically ways, already twenty years ago (for example [23]), also taking into account the action of dynamical friction by a uniform background of light stars [55]. The growing computing power recently allowed to build full 2D/3D viscous hydrodynamical and magnetohydrodynamic simulations of accretion systems on to close, or merging, binary SMBHs in GR, also regarding the modeling of electromagnetic radiation emitted by the surrounding gas or the predictions of individual feeded mini-accretion disks around each SMBH (for example $[6,11,15,42,46])$.

Pair of accreting SMBHs in single objects can be spatially resolved especially in radio bands if the separation is above some pc, while spatially unresolved binary SMBHs, can be probed with several indirect techniques (some examples in Fig. 5). Claims of quasi-periodic variability signatures could be in some cases explained ad unresolved binary SMBHs systems (OJ 287, PG 1302-102, 3C 345, PSO J334.2028+01.4075, AO 0235+16, 3C 273, and other), even if this is a very debated and controversial framework [26, 38, 39]. Other indirect evidences can be observed helical distorted radio jets with the jet-emitting secondary SMBH orbiting the primary with precession and nutations effects, and even jet reorientation in particular X-shaped radio galaxies (3C 345, NRAO 530/PKS 1730-13, 3C 120, 3C 66B, Mkn 501, and other,. see for example [29]). Another possibility is the observation of double-peaked broad lines (SDSS J0927+2943, SDSS J1316-1753, SDSS J150243.1+111557, PG 1302-102 where these are non-double but asymmetric), even if only a small fraction of all the double-peakers are real good binary candidates and there are very few confirmed as detections. Other evidences can be some cases of Tidal Disruption Events (for example SDSS J120136.02+300305.5, and [19]), and recoils effects for the newly formed SMBH by the anisotropic emission of GWs from the original coalescing binary SMBHs.

\section{The blazar OJ 287: a possible binary SMBH system}

Quasi periodic variations, postulated for long-term radio or optical flux light curves of few AGNs is a peculiar and controversial phenomenology. The well-known BL Lac object OJ 287 $(z=0.306$, a.k.a. PKS $0851+202$, S3 $0851+20$, Fig. 6) is a high-variable extragalactic source with 


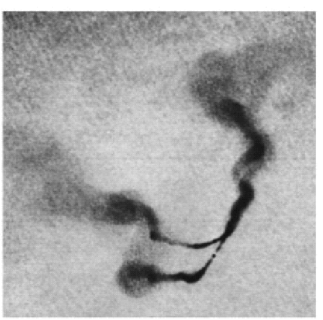

Dual jets $(3 \mathrm{C} 75$, a $7 \mathrm{kpc})$

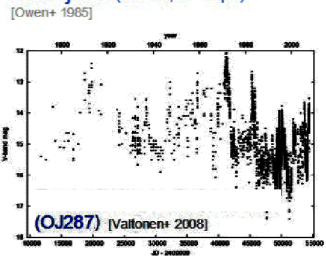

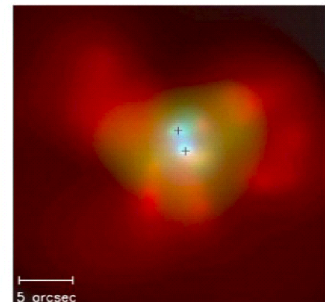

Dual X-ray sources (NGC 6240, a 1.5 kpc) [Komossa+ 2003]

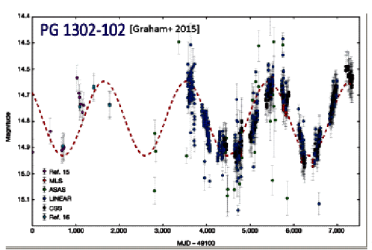

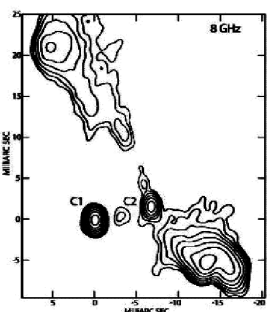

Binary radio sources $(0402+379$, a 7pc) [Rodriguez+ 2006]

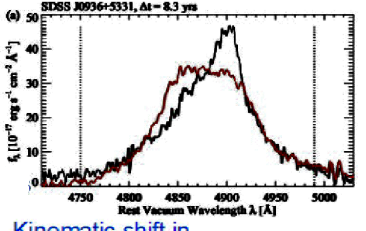

Kinematic shift in

multi-epoch observations [Shen+2013]

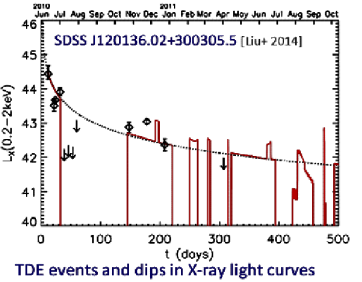

Quasi periodicity in light curves (still controversial topic)

Figure 5: Images and data for pair of accreting SMBH in single objects, spatially resolved if the separation is above some pc, here NGC 1128 (3C 75), NGC 6240, 4C +37.11 (B2 0402+37), and spatially unresolved binary SMBHs, here SDSS J0936+5331, OJ 287, PG 1302-102, PG 1553+113, SDSS J120136.02+300305.5. (adapted from [35, 27, 40, 41, 48, 18, 2, 28]).
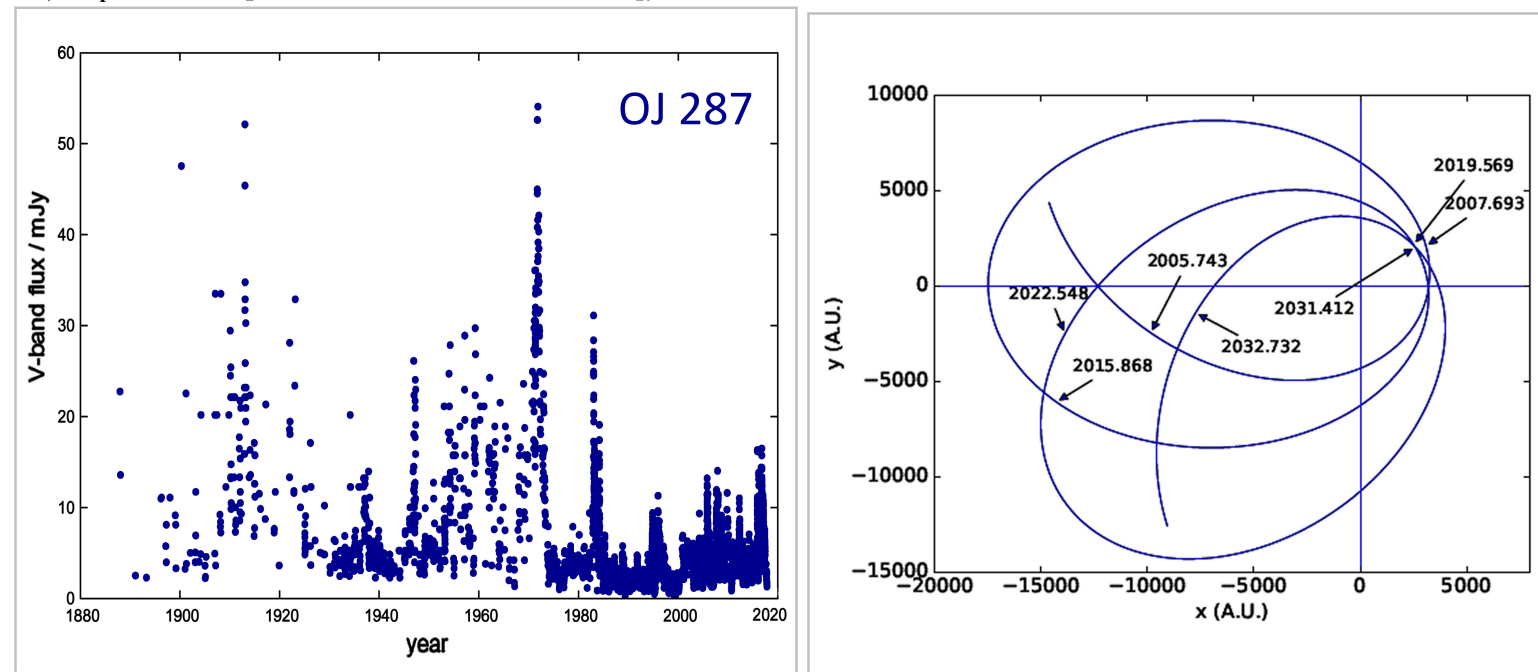

Figure 6: Left panel: about 130-year optical light curve of OJ 287. Right panel: Pictorial representation of the orbit of the secondary SMBH in OJ 287 from 2005 to 2033. The primary SMBH (Schwarzschild radius $R_{S} \simeq 362 \mathrm{AU}$ ) is situated at the origin with its accretion disk in the $y=0$ plane. The average distance of periastron is about $9 R_{S}$ and the binary is going to merge within 10000 years.

evidence for approximatively cyclical outbursts in the long-term history of the observed optical flux $[43,45,21,48]$. This blazar is one of the few cases where a large data set of intensive and extensive multifrequency light curve data is available. Quasi-periodic oscillations (QPOs, typically 1-10 Hz) of high-mass X-ray binaries, if produced in accretion flows coupled to the jet, could have a similar analogy in blazars on time-scales of years. QPOs in OJ 287 are non-strictly periodic, with double outburst events (separated by one/two years) and with orbital precession in a binary SMBHs system shrinking as clear signatures in the observed optical flux light curves (Fig. 6 and $[48,50,52])$.

Coordinated, both intensive and long-term, observing campaigns are active for OJ 287 since 
the beginning of 1990s: 1) the 1993-1997 OJ-94 monitoring project ${ }^{1}$; the 2005-2008 ENIGMA and WEBT projects with the related long-term and intensive multifrequency observing campaigns ${ }^{2}$; the 2006-2020 optical long-term monitoring project $^{3}$.

The postulated binary SMBH system at the center of OJ 287 has a sub-parsec separation and high-mass ratio $(100: 1)[43,48,52]$. Under this hypothesis the General Relativity orbital precession of the secondary $\mathrm{BH}$ naturally explains the approximatively periodic modulations of the optical light curve of OJ 287 [48, 52]. Einstein's General Relativity (GR) theory in strong-field conditions [48] was tested through light curve clocking. Such data also corroborated the sub-parsec binary SMBH hypothesis, where optical/UV/X-ray data have evidenced the separation between unboosted (non relativistically beamed) spectral component dominated by thermal bremsstrahlung emission from hot $\left(3 \times 10^{5}{ }^{\circ} \mathrm{K}\right)$ gas and the relativistically beamed in-jet, non-thermal, X-ray emission observed by XMM-Newton and Swift [9, 50, 49, 8, 56, 52]. Impact bremsstrahlung thermal emission dominating from UV to near-IR bands is also suggested by optical polarization data $[51,58,52]$. The loss in gravitational binding energy is caused by low frequency gravitational wave (GW) emission and the Lense-Thirring effect: the binary orbital plane precess, mainly due to the spin of the primary SMBH $[48,52]$. At the impact of the secondary on the accretion disk, a hot bubble of gas is extracted from the disk and expands at its internal sound speed. When it becomes optically thin during the expansion a burst of optical-UV radiation occurs.

The orbital motion is measurably different if the primary BH has no "hair" (no-hair theorem of GR) or if it has some "hair". The external gravitational field depends strictly only on the mass and the spin (net electric charge not expected in astronomical BHs). The occurrence of the optical outburst within the expected time window, using the high-mass ratio compact binary model is consistent with the no-hair theorem at the second post-Newtonian order, and confirms the energy losses by GW radiation in agreement within a few percents with the prediction by GR. The binary modeling of OJ 287 also indicated that the primary Kerr SMBH should spin approximately at quarter of the maximum spin rate allowed in GR, with a unique mathematical solution and prediction for impact flare outburst, that was observed on Dec. 2015. Its clocking is spin-sensitive, and this accurate light curve timing allowed us to constrain the Kerr spin parameter of the primary $\chi=(0.313 \pm 0.01)$ [52]. In this model the primary SMBH in OJ 287 is a black hole described by General Relativity with 30\% accuracy of the no-hair theorem and the outburst clocking also confirmed the energy loss by radiation of very low-frequency gravitational waves. The timescale from two galaxy merger to their central SMBH merger is usually of the order of $10^{8}-10^{9}$ years, and at the OJ 287 sub-parsec scale the time to merge is $<10^{5}$ years. The estimated parameters of the system are: SMBHs masses $m_{1}=(1.84 \pm 0.01) \times 10^{10} \mathrm{M}_{\odot} ; m_{2}=(1.40 \pm 0.03) \times 10^{8} \mathrm{M}_{\odot}$; $\Delta \phi=(39.1 \pm 0.1)^{\circ}$; primary Kerr SMBH spin $\chi=(0.313 \pm 0.01) ; e=0.66 \pm 0.01 ; q=1.0 \pm 0.8$ $[49,50,52]$.

The optical outburst epochs, clocked by the 130-year optical light curve dataset and the adopted binary SMBH model, are (in years): 1912.980 $\pm 0.020,1947.283 \pm 0.002,1957.095 \pm 0.025$, $1972.935 \pm 0.012,1982.964 \pm 0.0005,1984.125 \pm 0.01,1995.841 \pm 0.002,2005.745 \pm 0.015$,

\footnotetext{
${ }^{1}$ http://www.astro.utu.fi/research/oj94/

${ }^{2}$ http: //www.astro.utu.fi/OJ287MMVI/

${ }^{3}$ http: //www.as.up.krakow.pl/sz/oj287.html
} 

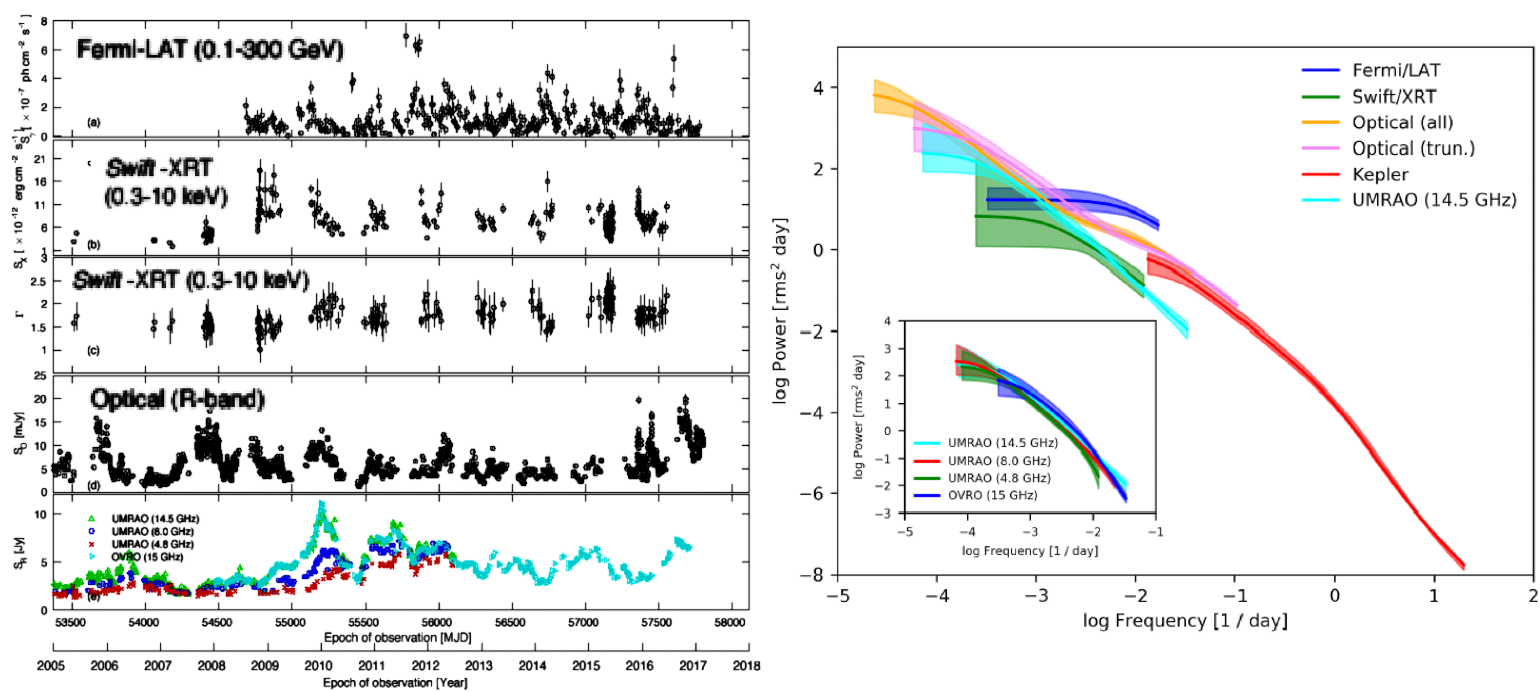

Figure 7: GeV gamma-rays (Fermi LAT), X-ray (Swift XRT), optical (many ground-based telescopes and the Kepler space telescope), radio-GHz (UMRAO and OVRO) flux light curves (also spectral photon index for XRT data) of OJ 287 from 2005 to 2018 and the relative PSDs. Data before 2005 (radio and optical) are not showed in the left plot. The analysis of the optical variability OJ 287 spanned 6 decades temporal frequencies, from 130 years to minute-scale sampling in Kepler data (adapted from [17]).

$2007.6915 \pm 0.0015,2015.875 \pm 0.025[13]$ and predicted 2019.724 (data analysis ongoing).

High-energy observations at X-ray bands (BeppoSAX, XMM-Newton, Suzaku, Swift, [31, 9, 52] ) and $\gamma$-ray bands (Fermi all-sky survey monitoring the source since Aug. 2008) have contributed to enlarge the physical picture on this blazar. An intensive and continuous three-month observing campaign of OJ 287 was performed by the Kepler space telescope, with 1 minute sampling at $>90 \%$ duty cycle and high S/N, during the K2 Campaign \#5 (Apr.27-Jul.13 2015, [58, 17]). No statistically significant QPO was detected in the range from minutes to 30 days.

Power density spectra (PDS) at GeV gamma-rays (Fermi LAT data), X-ray (Swift XRT data), optical (many ground-based telescopes and the Kepler space telescope data); radio-GHz by ground based radiotelescopes (UMRAO and OVRO) allowed the analysis of the optical variability OJ 287 across 6 decades temporal frequencies, thanks to Kepler and the 130-year optical historical light curves data set ([17] and Fig. 7). Light curves can be modeled in terms of continuous-time autoregressive moving average processes with a quantitative PDS evaluation. X-ray variability PDSs of OJ 287 are of a colored-noise (flicker/red-noise) ranging from tens of years to months, with no breaks, while the optical variability is of a colored-noise type ranging from 117 years down to hours. No significant signals of QPOs in the PDS (using the better sampled 40-year light curve since year 1970 can be a too short dataset for a 12-year significance analysis). The discrete Fourier transforms show anyway a $<3 \sigma$ peak for a strict 12-year period [17]. The PDS of the $E>100$ $\mathrm{MeV}$ gamma-ray light curve has hints for characteristic relaxation timescale of about 150 days. Above that scale the PDS consistent with uncorrelated (white) noise and below that scale with a colored-noise variability.

An independent result with long-term data can be obtained from radio-structure observations, usefully beyond the usual flux/polarization light curves. 120 Very Long Baseline Array (VLBA) observations at $15 \mathrm{GHz}$ of OJ 287 in the period from 1995 to 2017 are recently analyzed [5], 
showing that the radio jet is precessing on a time-scale of about 22 years and the data are consistent with a jet-axis rotation on a yearly time-scale, therefore with a combined motion of jet precession and jet-axis rotation. This can be in agreement with a deterministic, geometric mechanism: a precessing and nutating jet with accompanying viewing angle changes and consequent Doppler beaming variations for the radio bands.

There is not a strict theoretical upper limit to the mass grow of a black hole, it depends on its age and how fast is the accretion. The primary SMBH of OJ 287 is among the most massive known black hole in the universe $\left(m_{1}=(1.84 \pm 0.01) \times 10^{10} \mathrm{M}_{\odot}\right)$. OJ 287 seems to represent an indirect evidence for the existence of a compact binary spinning SMBH system emitting gravitational waves and, possibly, constituting an indirect test bench for General Relativity in strong field.

\section{References}

[1] Aartsen, M. G., Ackermann, M., Adams, J., et al. 2018, Science, 361, eaat1378

[2] Ackermann, M., Ajello, M., Albert, A., et al. 2015, ApJ Lett., 813, L41

[3] Arvanitaki, A., \& Dubovsky, S. 2011, Phys. Rev. D, 83, 044026

[4] Begelman, M. C., Blandford, R. D., \& Rees, M. J. 1980, Nature, 287, 307

[5] Britzen, S., Fendt, C., Witzel, G., et al. 2018, MNRAS, 478, 3199

[6] Bowen, D. B., Mewes, V., Campanelli, M., et al. 2018, ApJ Lett., 853, L17

[7] Cardoso, V., Dias, Ó. J. C., Hartnett, G. S., et al. 2018, Journ. Cosmology \& Astropart. Phys., 2018, 043

[8] Ciprini, S., Perri, M., Verrecchia, F., \& Valtonen M. 2015, ATel \#8401

[9] Ciprini, S., Raiteri, C. M., Rizzi, N., et al. 2007, Mem. SAIt, 78, 741

[10] Colpi, M. 2014, Space Science Rev., , 183, 189

[11] d'Ascoli, S., Noble, S. C., Bowen, D. B., et al. 2018, ApJ, 865, 140

[12] Dermer, C. D., \& Giebels, B. 2016, Comptes Rendus Physique, 17, 594

[13] Dey, L., Gopakumar, A., Valtonen, M., et al. 2019, Universe, 5, 108

[14] Dey, L., Valtonen, M. J., Gopakumar, A., et al. 2018, ApJ, 866, 11

[15] Farris, B. D., Duffell, P., MacFadyen, A. I., et al. 2015, MNRAS, 447, L80

[16] Ghisellini, G. 2000, in Recent Developments in General Relativity, B. Casciaro, D. Fortunato, M. Francaviglia, A. Masiello eds., Springer-Verlag, p. 5

[17] Goyal, A., Stawarz, Ł., Zola, S., et al. 2018, ApJ, 863, 175

[18] Graham, M. J., Djorgovski, S. G., Stern, D., et al. 2015, Nature, 518, 74

[19] , Hayasaki, K., \& Loeb, A. 2016, Scientific Reports, 6, 35629

[20] Hickox, R. C., \& Alexander, D. M. 2018, ARA\&A, 56, 625

[21] Hudec, R., Bašta, M., Pihajoki, P., \& Valtonen, M. 2013, A\&A, 559, A20

[22] Ivanov, P. B., Papaloizou, J. C. B., Paardekooper, S.-J., et al. 2015, A\&A, 576, A29 
[23] Ivanov, P. B., Papaloizou, J. C. B., \& Polnarev, A. G. 1999, MNRAS, 307, 79

[24] Kadler, M., Krauß, F., Mannheim, K., et al. 2016, Nature Physics, 12, 807

[25] Komossa, S. 2015, IAU General Assembly 29, 2254938

[26] Komossa, S. 2006, Memorie SAIt, 77, 733

[27] Komossa, S., Burwitz, V., Hasinger, G., et al. 2003, ApJ Lett., 582, L15

[28] Liu, F. K., Li, S., \& Komossa, S. 2014, ApJ, 786, 103

[29] Liu, F. K. 2004, MNRAS, 347, 1357

[30] Marscher, A. 2016, Galaxies, 4, 37

[31] Massaro, E., Giommi, P., Perri, M., et al. 2003, A\&A, 399, 33

[32] Mayer, L., Kazantzidis, S., Escala, A., et al. 2010, Nature, 466, 1082

[33] Mayer, L., Kazantzidis, S., Madau, P., et al. 2007, Science, 316, 1874

[34] Mikkola, S., \& Valtonen, M. J. 1990, ApJ, 348, 412

[35] Owen, F. N., O’Dea, C. P., Inoue, M., et al. 1985, ApJ Lett., 294, L85

[36] Padovani, P., Alexander, D. M., Assef, R. J., et al. 2017, The Astron. \& Astroph. Review, 25, 2

[37] Pihajoki, P., Valtonen, M., \& Ciprini, S. 2013, MNRAS, 434, 3122

[38] Rieger, F. M., 2004, ApJ Lett., 615, L5

[39] Rieger, F. M., 2007, Astrophys. \& Space Science, 309, 271

[40] Rodriguez, C., Taylor, G. B., Zavala, R. T., et al. 2006, ApJ, 646, 49

[41] Shen, Y., Liu, X., Loeb, A., et al. 2013, ApJ, 775, 49

[42] Shi, J.-M., Krolik, J. H., Lubow, S. H., et al. 2012, ApJ, 749, 118

[43] Sillanpää, A., Haarala, S., Valtonen, M. J., Sundelius, B., \& Byrd, G. G. 1988, ApJ, 325, 628

[44] Smith A., Bromm V., Loeb A., 2017, Astron. \& Geophys., 58, 3.22

[45] Takalo, L. O. 1994, Vistas in Astronomy, 38, 77

[46] Tang, Y., MacFadyen, A., \& Haiman, Z. 2017, MNRAS, 469, 4258

[47] Urry, C. M., \& Padovani, P. 1995, PASP, 107, 803

[48] Valtonen, M. J., Lehto, H. J., Nilsson, K., et al. 2008, Nature, 452, 851

[49] Valtonen, M., \& Ciprini, S. 2012, Mem. SAIt, 83, 219

[50] Valtonen, M. J., Ciprini, S., \& Lehto, H. J. 2012, MNRAS, 427, 77

[51] Valtonen, M., Zola, S., Gopakumar, A., et al. 2015, ATel \#8378

[52] Valtonen, M. J., Zola, S., Ciprini, S., et al. 2016, ApJ Lett., 819, L37

[53] Valtonen, M., Zola, S., Jermak, H., Ciprini, S., et al. 2017 Galaxies, 5, 83

[54] Vasiliev, E., Antonini, F., \& Merritt, D. 2014, ApJ, 785, 163

[55] Vecchio, A., Colpi, M., \& Polnarev, A. G. 1994, ApJ, 433, 733

[56] Verrecchia, F., Ciprini, S., Valtonen, M., \& Zola, S. 2016, ATel \#9709

[57] Wang, L., Greene, J. E., Ju, W., et al. 2017, ApJ, 834, 129

[58] Zola, S., Valtonen, M., Bhatta, G., Goyal, A., et al. 2016, Galaxies, 4, 41 